\title{
Morphological Characteristics of Grapevine Cultivars and Closed Contour Analysis with Elliptic Fourier Descriptors
}

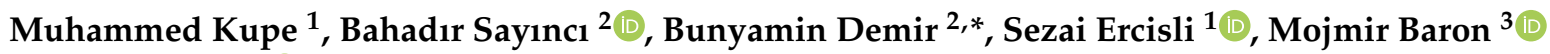 \\ and Jiri Sochor ${ }^{3}$ (I) \\ 1 Department of Horticulture, Faculty of Agriculture, Atatürk University, Erzurum 25240, Turkey; \\ muhammed.kupe@atauni.edu.tr (M.K.); sercisli@atauni.edu.tr (S.E.) \\ 2 Department of Mechanical Engineering, Faculty of Engineering, Mersin University, Mersin 33340, Turkey; \\ bsayinci@mersin.edu.tr \\ 3 Department of Viticulture and Enology, Faculty of Horticulture, Mendel University in Brno, Valticka 337, \\ 69144 Lednice, Czech Republic; mojmirbaron@seznam.cz (M.B.); jiri.sochor@mendelu.cz (J.S.) \\ * Correspondence: bd@mersin.edu.tr; Tel.: +90-324-361-00-01
}

Citation: Kupe, M.; Sayınc1, B. Demir, B.; Ercisli, S.; Baron, M.; Sochor, J. Morphological Characteristics of Grapevine Cultivars and Closed Contour Analysis with Elliptic Fourier Descriptors. Plants 2021, 10, 1350. https: / / doi.org/10.3390/ plants10071350

Academic Editors: Geza Bujdoso, Karoly Hrotko, Miljan Cvetkovic, Klara Cseke and Georgia Ntatsi

Received: 24 May 2021

Accepted: 29 June 2021

Published: 1 July 2021

Publisher's Note: MDPI stays neutral with regard to jurisdictional claims in published maps and institutional affiliations.

Copyright: (c) 2021 by the authors. Licensee MDPI, Basel, Switzerland. This article is an open access article distributed under the terms and conditions of the Creative Commons Attribution (CC BY) license (https:// creativecommons.org/licenses/by/ $4.0 /)$.

\begin{abstract}
Morphology is the most visible and distinct character of plant organs and is accepted as one of the most important tools for plant biologists, plant breeders and growers. A number of methods based on plant morphology are applied to discriminate in particular close cultivars. In this study, image processing analysis was used on 20 grape cultivars ("Amasya beyaz1", "Antep karası", "Bahçeli karası", "Çavuş", "Cevşen", "Crimson", "Dimrit", "Erenköy beyazı", "Hafızali",

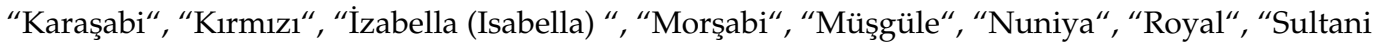
çekirdeksiz (Sultanina)", "Yalova incisi", "Yerli beyazv", "Yuvarlak çekirdeksiz") to classify them. According to image processing analysis, the longest and the greatest projected area values were observed in "Antep karası" cultivar. The "Sultani çekirdeksiz" cultivar had the least geometric mean diameter. The greatest sphericity ratios were observed in "Yerli beyaz", "Erenköy beyazi" and "Amasya beyazi" cultivars. According to principal component analysis, dimensional attributes were identified as the most significant source of variation discriminant grape cultivars from each other. Morphological differences between the cultivars were explained by sphericity and elongation variables. According to elliptic Fourier analysis (EFA) results, grape morphology largely looks like ellipse and sphere. However, there are some cultivars that look similar to a water drop. The cultivars with similar morphology were identified by a pair-wise comparison test conducted with the use of linear discriminant analysis, and they were presented in a scatter plot. According to cluster analysis, present grape cultivars were classified into seven sub-groups, which indicated great diversity.
\end{abstract}

Keywords: morphological analysis; projected area; dimensional analysis; contour analysis; sphericity

\section{Introduction}

Grape is one of the oldest horticultural crops. It is also one of the most cultivated horticultural plants, along with apple, citrus and banana. Total world grape production in 2018 was 79,000,000 metric tons, up by 6.5\% from 74,000,000 tons in 2017. China was the largest producer of grapes, with $13,397,000$ tons of production, followed, respectively, by Italy with $8,514,000$ tons, the USA with 6,891,000 tons, Spain with 6,673,00 tons, France with 6,198,000 tons and Turkey with 4,000,000 tons of production [1].

Worldwide, about $57 \%$ of grapes are used to make wine, $36 \%$ are consumed as fresh table grapes and $7 \%$ as dried grapes [2].

Common grapevines, Vitis vinifera L. are widely distributed, mainly in the temperate and subtropical regions in the world, including Mediterranean countries, Central and Southern Europe, to southwestern Asia. It is estimated that 10000 known grapevine varieties are distributed in grape growing areas throughout the world, and around 13 varieties dominate world production and cover more than one-third of the world's vineyard area [3]. 
The neighboring regions of the Caucasian area, including Turkey, have a long history of viticulture and possess a great diversity of local grape cultivars into different products. In Turkey, a large number of wild grapevines (Vitis vinifera L. subsp. sylvestris) and local cultivars are available [4]. The processes of cultivar selection and breeding started with wild grapes Vitis vinifera L. ssp. sylvestris Gmel. and included multiple introgression events from the wild to domestication [5], and it was initiated 8000 years ago in the South Caucasian area [6].

Previous archeological, palaeobotanical and historical studies confirm that grapevines were spread and cultivated for a long time in the Anatolia region in Turkey and have been an important part of civilizations established there [4,7]. In Anatolia, viticulture and wine-making have been developed for centuries by using local quite diverse grape cultivars. Those cultivars have been used for several purposes, such as for fresh consumption, drying, wine and spirit production and even for decorative and ornamental purposes $[5,6]$. During the last 3 decades, significant progress was achieved in the viticulture of Anatolia, and vineyards were diversified with foreign worldwide famous cultivars.

Turkey has more than 1500 national grape cultivars, of which 800 are genetically different. There are around 30 outstanding wine grape cultivars among them. The most suitable local Turkish grape cultivars for making wine are Bogazkere, Okuzgozu, Emir and Papazkarasi $[7,8]$.

Local grape cultivars are mainly grown in old vineyards located in ancient settlements and homesteads. There are also different growing systems, including suspended and creeping cultivations. The local grape cultivars differ from each other by their morphological characteristics and sizes of the bunches and berries; phenology; time of harvest; productivity and quality indices [9-11]. Local grape cultivars are essential to sustain crop diversity and can also be essential for food, nutrition and economic security of many people-particularly smallholder farmers and farming communities in rural and marginal areas. The diversity in local grapes can provide assurance against crop failure and offer special materials for traditional local cuisines and specific dietary requirements. Furthermore, these diverse grape cultivars are an important source of locally adapted genes for the improvement of the new grape cultivars [12,13].

Turkey's natural and ecological endowments are favorable for producing grapes. The grape industry could be an important part of agriculture from the point of view of employment and value creation. Due to its role in tourism and its opportunities for export, the industry can be regarded as one of the industries to be strategically developed. In addition, many regions of Turkey are rich in valuable local grapevine varieties which have not been explored and fully characterized yet [14].

In each grape growing country, there were numerous local varieties that contribute to world grapevine diversity $[15,16]$. Within a grape variety, significant clonal variability was evident $[17,18]$. Thus, the definition and the identification of varieties are of considerable scientific and practical importance in modern viticulture and ampelography [19-22].

Grape berries belonging to different cultivars show considerable diversity in berry morphology, in particular for berry size, color and shape. Berry size, color and shape attributes are of primary importance in the perceived quality and overall acceptability to consumer preference $[10,11]$. Correct cultivar identification in grapes is important to grape growers, regulatory authorities and winemakers. More recently, with the advertisement on grape berry composition, an increasing interest in new grapevine plantations has occurred, and there is a need to ensure trueness to the type of grape-planting material. Mistakes on variety trueness may result in significant financial costs not only for growers but also related industry [23]. For a long time, traditional grapevine variety identification was made by visual inspection of the grapevine that is known as ampelography. However, the use of ampelography in variety identification in grapevine varieties does not give an exact result, and some variability in descriptor definition may occur due to environmental conditions, cultural practices and genetic variations. For example, the same grape variety shows size, shape and color variability on berries and bunches in different environments. Health 
status, including diseases, can make classification more complex [24-26]. Ampelographic descriptions for a variety vary slightly according to the interpretation of the observer as well. In addition, it is important to establish relativity with the descriptors used, particularly in trying to distinguish between similar varieties [9-11]. In general, the berry shape index was used for descriptors in grapevine cultivars, but fruit shape is a three-dimensional characteristic and must be defined using pleiotropic explanatory variables rather than a simple/single index [27].

Recently, some sophisticated methods, including multivariate analysis [28], artificial neural network [29], DNA marker technologies including SSR and SNP [30,31] and Elliptic Fourier analysis [32], have been using in grapevine for ampelographic data processing and variety trueness. All these methods could be efficiently used to determine the differences or synonymy of grapevine genotypes.

Elliptic Fourier analysis (EFA) is gained more importance with the improvements of computer performance along with decreases in the cost of digital imaging hardware and software more recently. Thus the method widely contributed digital image processing applications for agriculturally relevant morphological analyses in different crop species [27,33-35]. These studies indicated that EFA provided an excellent tool for shape discrimination of several agricultural products. However, studies on EFA on grapevine cultivars are very limited in the literature. The main approach of this method is better defining the complex shape of fruits, etc. The method requires a set of coordinate values or descriptors obtained in a Fourier analysis [27]. This method determines the overall shape based on image data by first transforming coordinate information regarding the image contours into EFDs (Elliptic Fourier Descriptors), which are then summarized by a PCA (Principal Component Analysis). Analyses based on EFDs and PCA have been completed using the SHAPE program [36].

This study was conducted to investigate the size and shape features describing the physical attributes of 20 grapevine cultivars and to reveal shape distinctions with Elliptic Fourier descriptors modeling the closed contour of the cultivars.

\section{Materials and Methods}

\subsection{Locations of Grape Cultivars}

The study was conducted at the commercial farm Kemerhisar, Nigde province in middle Turkey during 2020. The geographical coordinates of the experiment location were $37.8318^{\circ} \mathrm{N}$ latitude and $34.6001^{\circ} \mathrm{E}$ longitude, and $1120 \mathrm{~m}$ elevation above sea level. All 20 grape cultivars were harvested from a 40-year-old wire-trained vineyard on the date of 12.09.2020. On the same day of harvest, samples were transported in cold-chain to Advanced Technology Research and Implementation Center of Mersin University. Daily irrigations from May to September and other management practices (herbicide and fertilizer applications and pruning) were conducted according to the farm manager's criteria. Herbicides were periodically applied between rows to control weeds. Vines were pruned with shears in February each year. The berry skin color of grapevine cultivars is given in Table 1.

\subsection{Imaging System and Sampling}

Twenty grape cultivars used in present experiments are presented in Figure 1. For analyses, 40 berry samples were randomly selected from each cultivar. Berry samples were placed on white fiberglass plate in $4 \times 5$ matrix array in 2 groups. Cylinder-formed plastic supports were used to fixate samples in both horizontal and vertical orientation. Grape cultivars were imaged with the use of Nikon D90 model digital camera and image files with *.tiff extension were recorded. Imaging system is presented in Figure 2. Artificial lighting was provided beneath the fiberglass plate to clarify the contours of berry samples [37]. Transparent surfaces were used to provide a contrast between plate and berry color. Digital camera was mounted and fixed on a tripod. Imaging was performed $56 \mathrm{~cm}$ above the samples. A shutter release cord was used to prevent vibrations while taking the images. 
Grape samples were imaged at both horizontal and vertical orientations. A millimetric ruler was placed by the grape samples to convert pixel units into millimeters.

Table 1. External (skin) berry color of cultivars.

\begin{tabular}{cc}
\hline Cultivars & Berry Skin Color \\
\hline Amasya beyazı & Green-yellow \\
\hline Antep karası & Black \\
\hline Bahçeli karası & Black \\
\hline Çavuş & Green-yellow \\
\hline Cevşen & Green-yellow \\
\hline Crimson & Dark red \\
\hline Dimrit & Purple-black \\
\hline Erenköy beyazı & Green-yellow \\
\hline Hafizali & Green-yellow \\
\hline Karaşabi & Black \\
\hline Kırmızı & Dark red \\
\hline Izabella (İsabella) & Dark purple-black \\
\hline Morşabi & Purple \\
\hline Müşgüle & Green-yellow \\
\hline Nuniya & Green-yellow \\
\hline Royal & Black \\
\hline Yuvarlak çekirdeksiz & Green-yellow \\
\hline Yalova incisi & Green-yellow \\
\hline Yerli beyaz & Green-yellow \\
\hline çekirdeksiz (Sultanina) & Green-ellow \\
\hline
\end{tabular}

\subsection{Morphology and Dimensional Attributes}

SigmaScan ${ }^{\circledR}$ Pro 5.0 software was used to determine morphology and dimensional attributes of the grape cultivars. For image processing, $0-255$ threshold range was applied to monochrome images and dimension analysis was automatically performed. Calibration was made over the ruler to convert pixel units into millimeters. With the present analyses, length $(\mathrm{L}, \mathrm{mm})$, width $(\mathrm{W}, \mathrm{mm})$, thickness $(\mathrm{T}, \mathrm{mm})$, projection area $\left(\mathrm{PA}, \mathrm{mm}^{2}\right)$, equivalent diameter $(\mathrm{ED}, \mathrm{mm})$, perimeter $(\mathrm{P}, \mathrm{mm})$ and circularity $(\mathrm{C})$ were automatically measured. Dimensional and area measures are presented in Figure 3 and equations used in calculations are provided in Table 2 .

\subsection{Elliptic Fourier Analysis}

For Elliptic Fourier analyses (EFA), 40 grape image files were used for each cultivar. EFA analysis was conducted in different phases with the use of MORPHOLOGY (version 1.03) software [36]. In phase I, contours of a closed morphology were defined. In phase II, $x$ and $y$ coordinates of the points on contoured curve were determined. In phase III, coordinate values were converted into mathematical functions. In phase IV, function coefficients were obtained [43]. For function coefficients, analyses were conducted over 20 harmonics. Each harmonic produces four Fourier coefficients (an, bn, cn and dn). The an and bn coefficients correspond to $\mathrm{x}$ coordinate and $\mathrm{cn}$ and dn coefficients to $\mathrm{y}$ coordinate of the curve [44,45].

For image processing, grape images were converted in 24 -bit ${ }^{*}$.bmp format. Four modules were used to obtain morphological data. In module I (ChainCoder), image 
processing and morphology contour codes were generated. In module II (Chc2Nef), contour codes were normalized and Elliptic Fourier descriptors were obtained. In module III (PrinComp), descriptors were subjected to PC analysis and PC scores were obtained. In module IV (PrinPrint), morphology variations of grape image contours were visualized.

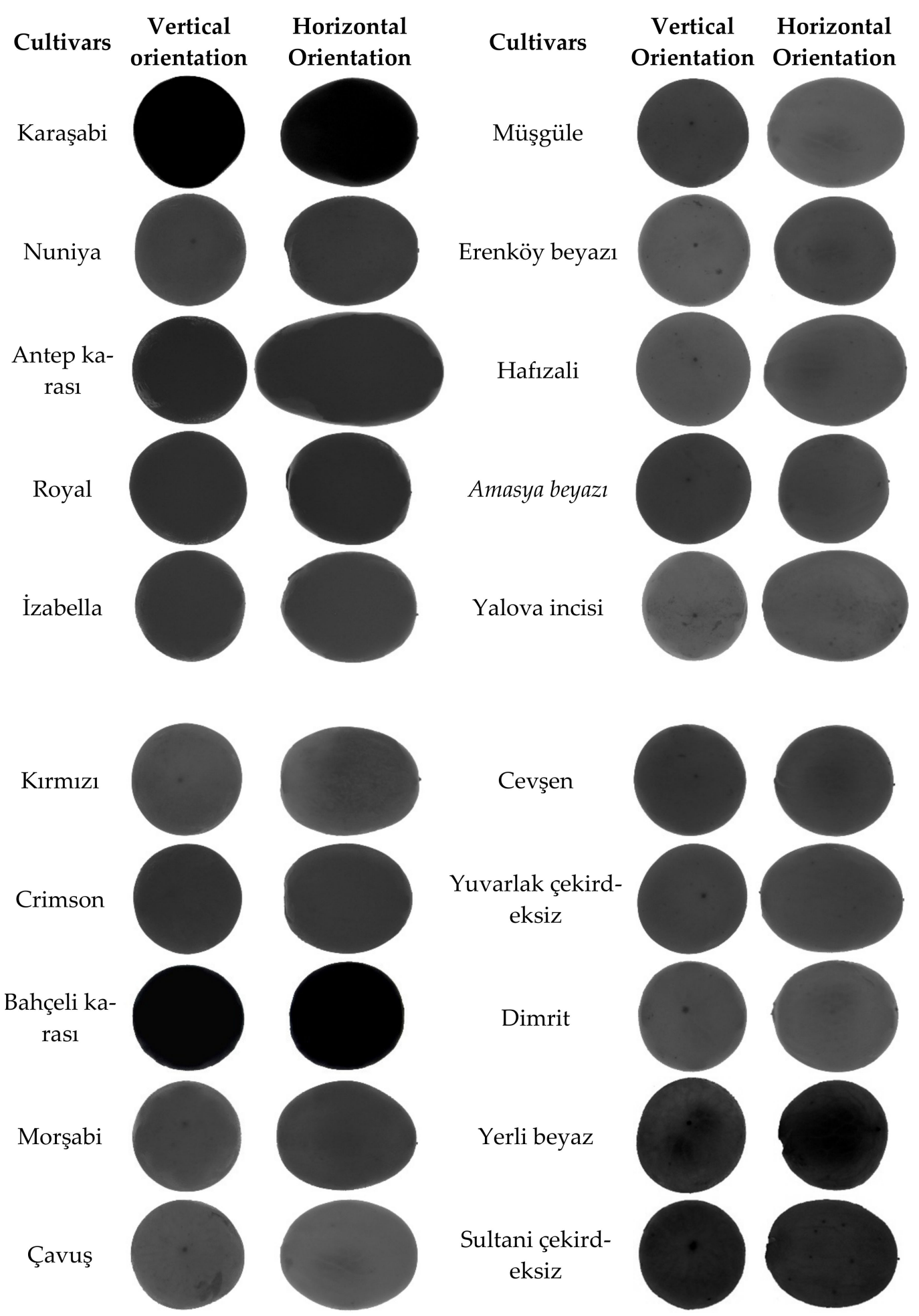

Figure 1. Grapevine cultivars. 


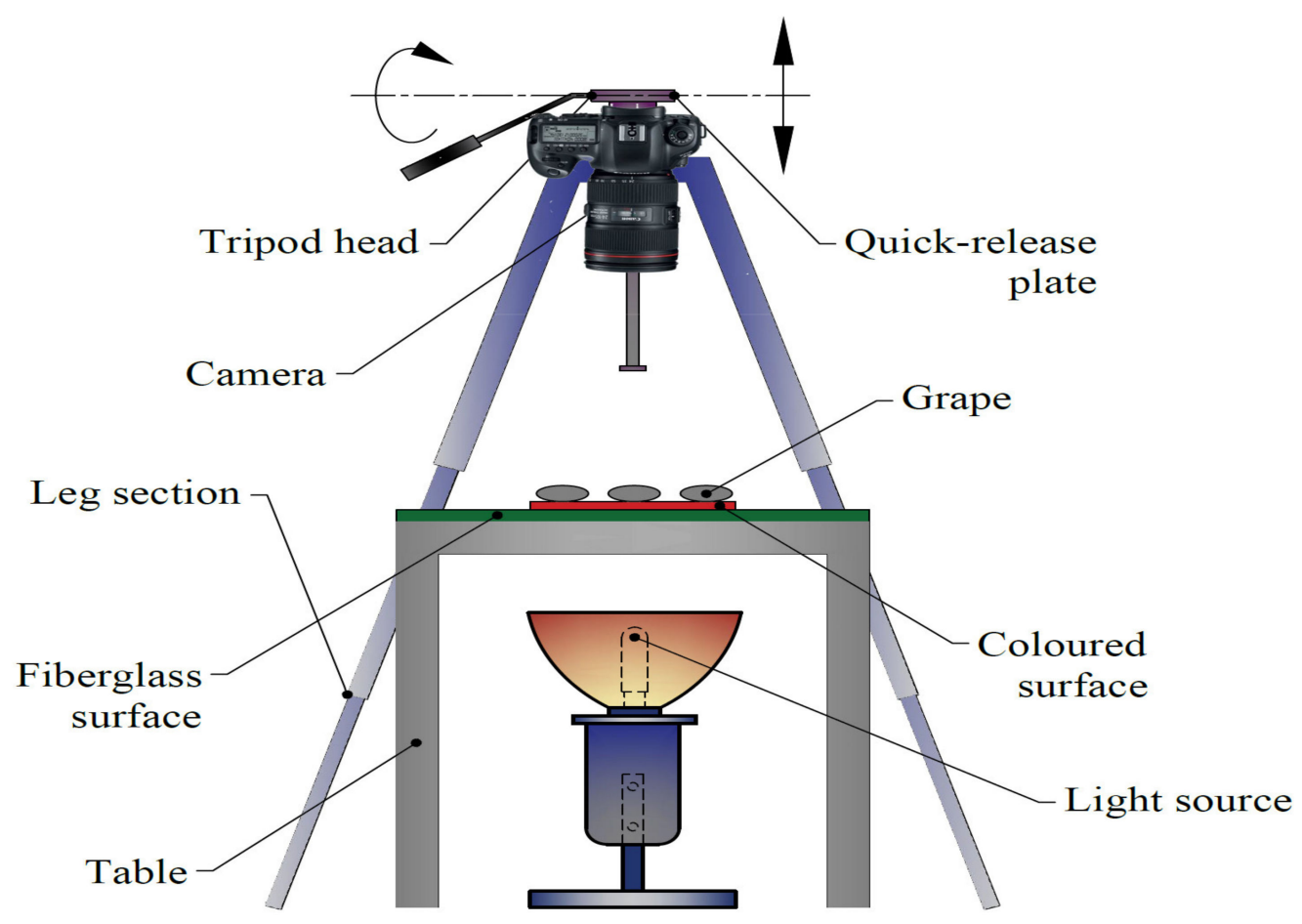

Figure 2. Image acquisition system.

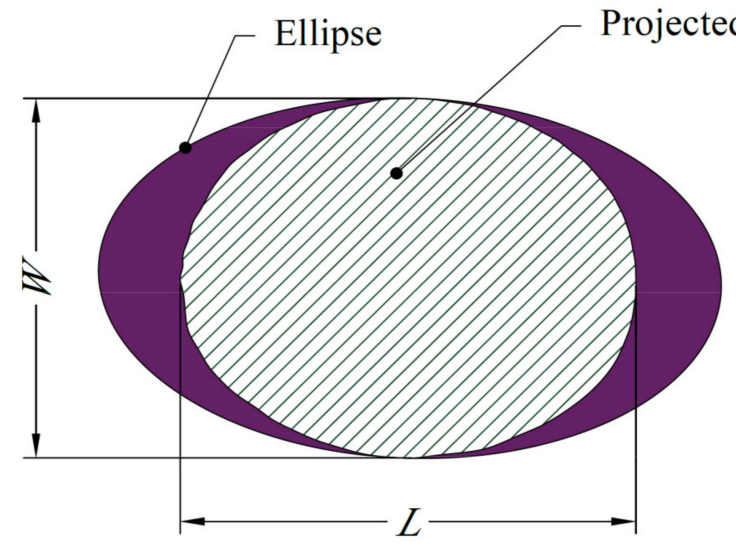

Horizontal orientation

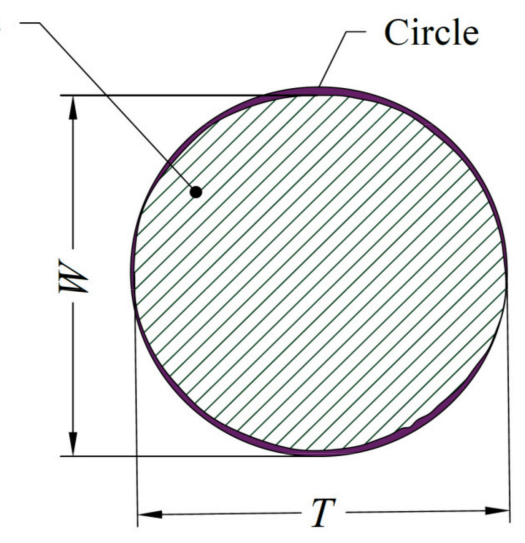

Vertical orientation

Figure 3. Size and area measurements of the grape cultivars.

Table 2. Equations used for morphology and dimensional attributes of the grape cultivars.

\begin{tabular}{ccc}
\hline $\begin{array}{c}\text { Morphological- } \\
\text { Dimensional Attributes }\end{array}$ & Equations & References \\
\hline & $A R_{h}=L / W$; for hor. orientation & \\
Aspect ratio $(A R)$ & $A R_{v}=W / T ;$ for \\
& vertical orientation & \\
Geometric mean diameter $\left(D_{g}, \mathrm{~mm}\right)$ & $D_{g}=\sqrt[3]{(L \cdot W \cdot T)}$ & {$[39]$} \\
Sphericity $(\varphi, \%)$ & $\varphi=\left(D_{g} / L\right) \cdot 100$ & {$[40]$} \\
Volume $\left(V, \mathrm{~mm}^{3}\right)$ & $V=(\pi / 6) \cdot(L \cdot W \cdot T)$ & Ellipse volume \\
Surface area $\left(S A, \mathrm{~mm}^{2}\right)$ & $S A=\pi \cdot D_{g}^{2}$ & {$[41]$} \\
Circularity $(C)$ & $C=4 \cdot \pi \cdot P A / P^{2}$ & {$[42]$} \\
\hline
\end{tabular}




\subsection{Statistical Assessments}

Each variable of morphological characteristics of grape cultivars was subjected to analysis of variance (ANOVA) and significant means were compared with the use of Duncan's test at 5\% significance level. All variables of morphology and dimensional attributes were subjected to Principal Component Analysis (PCA) and differences between the cultivars were presented in scatter plots based on component scores. With PC analysis, significant variables revealing morphology and dimensional differences of the cultivars were identified and ordered. SPSS 20.0 software was used for statistical analyses.

Normalized contour codes by Elliptic Fourier analysis (EFA) were subjected to multivariate variance analysis (MANOVA) with the use of PAST v.4.02 software. Morphological differences between grape cultivars were explained by Hotelling's pair-wise comparison tests, including verified Bonferroni values and Mahalanobis distances. In linear discriminant analysis conducted with the use of principal component (PC) scores, functions revealing morphology differences of the grape cultivars were determined and similarity relationships were presented in scatter plots. Such similarities were also put forth by hierarchical cluster analysis with the use of Euclidean similarity index and the grape cultivars, with morphology similarities presented in a dendrogram.

\section{Results and Discussion}

\subsection{Basic Morphology and Dimensional Attributes Measured at Horizontal and Vertical Orientations}

Morphology and dimensional attributes of the grape cultivars measured at horizontal and vertical orientations are provided in Table 3. Projection areas measured at both orientations varied in a broad range. Such a case revealed that there were significant physical differences between the grape cultivars. The "Antep karasi", "Hafızali" and "Royal" cultivars had the greatest projected areas. Equivalent diameter means were greater at horizontal orientation than at vertical orientation. Increased perimeters were observed in cultivars with the greatest projected area. The greatest elongation average was measured at horizontal orientation. The morphology looks like a full circle as the elongation ratio approaches 1 . The lowest elongation ratios were observed in "Yerli beyaz", "Erenköy beyazı" and "Amasya beyazi" cultivars. Previously, Ekhvaia and Akhalkatsi [46] and Leão et al. [47] studied grape genotypes based on berry dimensions and reported high variability. Khadivi-Khub et al. [48] analyzed grape germplasm from Iran based on fruit dimensions and they revealed a significant difference among the evaluated grape cultivars. Kok et al. [49] investigated dimensional attributes of eight grape cultivars in western Turkey and found that berry dimensions were quite variable among eight grape cultivars. Previous studies indicated that grape berry dimensions are cultivar-dependent, yet are affected by numerous factors, including gibberellin treatments, girdling, soil type, irrigation, rootstock and the weather, etc. [50-52]. Esgici et al. [53] reported the length, width and thickness of "Şire" grapes, respectively, as $16.16 \mathrm{~mm}, 15.43 \mathrm{~mm}$ and $15.51 \mathrm{~mm}$. Present findings of 20 grape cultivars complied with the values of previous studies.

\subsection{Basic Morphology and Dimensional Attributes of the Grape Cultivars}

The greatest length average was observed in "Antep karası" and the lowest in "Bahçeli karası" cultivar (Table 4). The greatest geometric mean diameter, surface area and volume averages were observed in "Antep karas", "Royal" and "Hafızali" cultivars. In terms of morphology, the grape cultivars with the closest morphology to circle were identified as "Yerli beyaz", "Erenköy beyazı" and "Amasya beyazı". Elongation at horizontal orientation designates the longness or shortness of the cultivars. Therefore, the circularity average of 1 measured at horizontal orientation indicates that the morphology is a full circle. The greatest circularity averages were observed in "Bahçeli karası" and "Royal" and the lowest in "Antep karası" cultivars. Khodaei and Akhijahani [54] reported geometric mean diameters of "Rasa" grapes based on moisture contents as between 9.20 and $14.74 \mathrm{~mm}$ and sphericity values as between 61 and $89 \%$. Esgici et al. [53] reported geometric mean 
diameter of "Şire" grape as $14.33 \mathrm{~mm}$ and sphericity value as 97.1\%. In this sense, "Rasa" and "Şire" grape cultivars were similar with "Bahçeli karası" and "Sultani çekirdeksiz (Sultanina)" cultivars.

Table 3. Basic morphology and dimensional attributes measured at horizontal and vertical orientations.

\begin{tabular}{|c|c|c|c|c|c|c|c|c|}
\hline \multirow[b]{2}{*}{ Varieties } & \multicolumn{4}{|c|}{ Horizontal Orientation } & \multicolumn{4}{|c|}{ Vertical Orientation } \\
\hline & $\begin{array}{l}\text { Projected Area } \\
\qquad\left(\mathrm{mm}^{2}\right)\end{array}$ & $\begin{array}{l}\text { Feret Diameter } \\
(\mathrm{mm})\end{array}$ & $\begin{array}{l}\text { Perimeter } \\
(\mathrm{mm})\end{array}$ & Aspect Ratio & $\begin{array}{l}\text { Projected Area } \\
\left(\mathrm{mm}^{2}\right)\end{array}$ & $\begin{array}{c}\text { Feret Diameter } \\
(\mathrm{mm})\end{array}$ & $\begin{array}{l}\text { Perimeter } \\
(\mathrm{mm})\end{array}$ & Aspect Ratio \\
\hline Amasya beyazı & $371.2 \pm 28.0 \mathrm{~d}, *$ & $21.73 \pm 0.81^{\mathrm{d}}$ & $73.68 \pm 2.94^{\mathrm{d}}$ & $1.072 \pm 0.042 \mathrm{klm}$ & $356.8 \pm 31.0^{\mathrm{b}}$ & $21.30 \pm 0.91 \mathrm{~b}$ & $71.31 \pm 3.11 \mathrm{~b}$ & $1.028 \pm 0.016^{\mathrm{f}}$ \\
\hline Antep karası & $516.1 \pm 47.9^{\mathrm{a}}$ & $25.61 \pm 1.19^{\mathrm{a}}$ & $89.21 \pm 4.21$ a & $1.557 \pm 0.103^{\mathrm{a}}$ & $347.6 \pm 37.6 \mathrm{bc}$ & $21.01 \pm 1.14 \mathrm{bc}$ & $70.52 \pm 4.01 \mathrm{~b}$ & $1.057 \pm 0.041 \mathrm{a}$ \\
\hline Bahçeli karası & $150.8 \pm 14.2 \mathrm{~m}$ & $13.84 \pm 0.65^{\circ}$ & $46.51 \pm 2.26^{\circ}$ & $1.100 \pm 0.035^{\mathrm{j}}$ & $136.4 \pm 13.7^{j}$ & $13.16 \pm 0.65^{j}$ & $44.73 \pm 2.30^{j}$ & $1.032 \pm 0.017$ cdef \\
\hline Çavuş & $345.3 \pm 37.6 \mathrm{e}$ & $20.94 \pm 1.13 \mathrm{e}$ & $71.34 \pm 4.15^{\mathrm{e}}$ & $1.103 \pm 0.044 \mathrm{j}$ & $316.0 \pm 40.4^{\mathrm{d}}$ & $20.02 \pm 1.27 \mathrm{~d}$ & $68.00 \pm 4.06^{\mathrm{c}}$ & $1.036 \pm 0.018$ bcdef \\
\hline Cevşen & $297.6 \pm 16.2^{\mathrm{h}}$ & $19.46 \pm 0.53 \mathrm{~h}$ & $65.97 \pm 1.83 \mathrm{~h}$ & $1.095 \pm 0.039 \mathrm{jkl}$ & $278.3 \pm 17.3^{\mathrm{f}}$ & $18.81 \pm 0.58^{\mathrm{f}}$ & $63.32 \pm 1.99 \mathrm{e}$ & $1.034 \pm 0.017$ cdef \\
\hline Crimson & $276.4 \pm 29.3^{\mathrm{i}}$ & $18.73 \pm 0.98^{\mathrm{j}}$ & $63.63 \pm 3.38^{\mathrm{ji}}$ & $1.218 \pm 0.056 \mathrm{~g}$ & $229.1 \pm 22.4 \mathrm{~h}$ & $17.06 \pm 0.83^{\mathrm{h}}$ & $57.16 \pm 2.71 \mathrm{~h}$ & $1.032 \pm 0.017$ cdef \\
\hline Dimrit & $256.6 \pm 18.2^{j}$ & $18.06 \pm 0.64 \mathrm{k}$ & $61.27 \pm 2.10 \mathrm{k}$ & $1.100 \pm 0.042 \mathrm{j}$ & $233.7 \pm 17.5^{\mathrm{h}}$ & $17.24 \pm 0.64 \mathrm{~h}$ & $58.52 \pm 2.07 \mathrm{~g}$ & $1.036 \pm 0.018$ bcdef \\
\hline Erenköy beyazı & $289.9 \pm 18.9 \mathrm{hi}$ & $19.20 \pm 0.63 \mathrm{hi}$ & $65.40 \pm 2.42$ hi & $1.069 \pm 0.028 \mathrm{~lm}$ & $277.6 \pm 20.3 \mathrm{f}$ & $18.79 \pm 0.68^{\mathrm{f}}$ & $63.55 \pm 2.44 \mathrm{e}$ & $1.027 \pm 0.016^{\mathrm{f}}$ \\
\hline Hafizali & $422.8 \pm 37.6^{b}$ & $23.18 \pm 1.04 \mathrm{~b}$ & $79.03 \pm 3.88^{b}$ & $1.285 \pm 0.058 \mathrm{de}$ & $337.1 \pm 30.3^{c}$ & $20.70 \pm 0.93 c$ & $69.14 \pm 3.09^{c}$ & $1.031 \pm 0.017$ cdef \\
\hline Karaşabi & $320.1 \pm 27.4 \mathrm{~g}$ & $20.17 \pm 0.87 \mathrm{~g}$ & $68.22 \pm 3.08 \mathrm{~g}$ & $1.245 \pm 0.059 \mathrm{f}$ & $265.5 \pm 24.2 \mathrm{~g}$ & $18.37 \pm 0.85 \mathrm{~g}$ & $61.40 \pm 2.82 \mathrm{f}$ & $1.042 \pm 0.027 \mathrm{bcd}$ \\
\hline Kirmızi & $282.7 \pm 25.3^{\mathrm{i}}$ & $18.95 \pm 0.83$ ij & $64.40 \pm 2.96 \mathrm{ji}$ & $1.277 \pm 0.061 \mathrm{de}$ & $223.1 \pm 19.9^{\mathrm{h}}$ & $16.84 \pm 0.74 \mathrm{~h}$ & $56.47 \pm 2.35 \mathrm{~h}$ & $1.031 \pm 0.020 \mathrm{def}$ \\
\hline İzabella & $262.6 \pm 27.5^{j}$ & $18.26 \pm 0.95^{\mathrm{k}}$ & $61.92 \pm 3.27 \mathrm{k}$ & $1.198 \pm 0.058 \mathrm{hg}$ & $225.7 \pm 27.0^{\mathrm{h}}$ & $16.92 \pm 1.00^{\mathrm{h}}$ & $56.73 \pm 3.34 \mathrm{~h}$ & $1.036 \pm 0.024$ bcdef \\
\hline Morşabi & $327.5 \pm 29.0 \mathrm{fg}$ & $20.40 \pm 0.91 \mathrm{fg}$ & $69.44 \pm 3.29 \mathrm{fg}$ & $1.266 \pm 0.057 \mathrm{ef}$ & $262.3 \pm 25.6 \mathrm{~g}$ & $18.25 \pm 0.90 \mathrm{~g}$ & $61.22 \pm 2.97 \mathrm{f}$ & $1.030 \pm 0.016$ ef \\
\hline Müşgüle & $338.6 \pm 22.6$ ef & $20.75 \pm 0.69$ ef & $70.65 \pm 2.43$ ef & $1.146 \pm 0.052^{\mathrm{i}}$ & $299.8 \pm 18.6 \mathrm{e}$ & $19.53 \pm 0.60 \mathrm{e}$ & $66.00 \pm 1.86 \mathrm{~d}$ & $1.034 \pm 0.016$ cdef \\
\hline Nuniya & $242.5 \pm 25.8 \mathrm{k}$ & $17.55 \pm 0.94 \mathrm{ij}$ & $59.31 \pm 3.19^{1}$ & $1.180 \pm 0.050 \mathrm{~h}$ & $208.1 \pm 23.8^{\mathrm{i}}$ & $16.25 \pm 0.93^{\mathrm{i}}$ & $54.56 \pm 3.12^{\mathrm{i}}$ & $1.040 \pm 0.026$ bcde \\
\hline Royal & $410.5 \pm 43.0 \mathrm{bc}$ & $22.83 \pm 1.19 \mathrm{bc}$ & $76.80 \pm 3.96^{c}$ & $1.097 \pm 0.055$ ij & $379.7 \pm 43.3^{\mathrm{a}}$ & $21.95 \pm 1.23 \mathrm{a}$ & $73.26 \pm 4.06 \mathrm{a}$ & $1.046 \pm 0.020 \mathrm{~b}$ \\
\hline Sultani çekirdeksiz & $159.7 \pm 9.8 \mathrm{~m}$ & $14.25 \pm 0.44 \mathrm{n}$ & $48.99 \pm 1.72 \mathrm{n}$ & $1.326 \pm 0.070 \mathrm{c}$ & $120.6 \pm 8.4 \mathrm{k}$ & $12.38 \pm 0.43 \mathrm{k}$ & $42.12 \pm 1.67 \mathrm{k}$ & $1.042 \pm 0.025 b c$ \\
\hline Yalova incisi & $401.1 \pm 50.2^{\mathrm{c}}$ & $22.56 \pm 1.40^{\mathrm{c}}$ & $77.63 \pm 4.90^{\mathrm{c}}$ & $1.415 \pm 0.080 \mathrm{~b}$ & $285.4 \pm 40.5 \mathrm{f}$ & $19.02 \pm 1.34^{\mathrm{f}}$ & $65.51 \pm 4.99 \mathrm{~d}$ & $1.034 \pm 0.017$ cdef \\
\hline Yerli beyaz & $201.3 \pm 17.2^{1}$ & $16.00 \pm 0.68 \mathrm{~m}$ & $55.09 \pm 2.53 \mathrm{~m}$ & $1.055 \pm 0.029 \mathrm{~m}$ & $201.9 \pm 15.6^{\mathrm{i}}$ & $16.02 \pm 0.62^{\mathrm{i}}$ & $54.26 \pm 2.10^{\mathrm{i}}$ & $1.033 \pm 0.018$ cdef \\
\hline Yuvarlak çekirdeksiz & $286.2 \pm 20.8 \mathrm{hi}$ & $19.08 \pm 0.69$ hij & $65.17 \pm 2.49 \mathrm{hi}$ & $1.297 \pm 0.056^{\mathrm{d}}$ & $225.4 \pm 16.6^{\mathrm{h}}$ & $16.93 \pm 0.62 \mathrm{~h}$ & $56.86 \pm 2.02 \mathrm{~h}$ & $1.037 \pm 0.018$ bcdef \\
\hline Mean \pm SD & $308.0 \pm 92.0$ & $19.58 \pm 2.98$ & $66.68 \pm 10.38$ & $1.205 \pm 0.140$ & $260.5 \pm 71.9$ & $18.03 \pm 2.59$ & $60.73 \pm 8.60$ & $1.036 \pm 0.022$ \\
\hline Min-max & $119.8-619.4$ & $12.35-28.08$ & $41.41-98.43$ & $1.008-1.782$ & $106.9-518.4$ & $11.67-25.69$ & $39.2-85.49$ & $1.000-1.216$ \\
\hline
\end{tabular}

*: Means followed by the same letter in the same column are not significantly different based on Duncan's test at $5 \%$ significance level.

Table 4. Basic morphology and dimensional attributes of the grape cultivars.

\begin{tabular}{|c|c|c|c|c|c|c|c|c|}
\hline Varieties & $\begin{array}{l}\text { Length } \\
(\mathrm{mm})\end{array}$ & $\begin{array}{l}\text { Width } \\
(\mathrm{mm})\end{array}$ & Thickness (mm) & $\begin{array}{l}\text { Geometric Mean } \\
\text { Diameter }(\mathrm{mm})\end{array}$ & $\begin{array}{c}\text { Sphericity } \\
(\%)\end{array}$ & $\begin{array}{l}\text { Surface Area } \\
\left(\mathrm{cm}^{2}\right)\end{array}$ & $\begin{array}{l}\text { Volume } \\
\left(\mathrm{cm}^{3}\right)\end{array}$ & Circularity \\
\hline Amasya beyazı & $22.67 \pm 0.92 \mathrm{de}^{*}$ & $21.17 \pm 0.96^{b}$ & $21.46 \pm 0.93 \mathrm{~b}$ & $21.75 \pm 0.84^{\mathrm{c}}$ & $96.0 \pm 2.0^{\mathrm{a}}$ & $14.89 \pm 1.16^{\mathrm{c}}$ & $5.413 \pm 0.638^{c}$ & $0.858 \pm 0.020 \mathrm{bcc}$ \\
\hline Antep karası & $31.94 \pm 1.69^{\mathrm{a}}$ & $20.56 \pm 1.22^{c}$ & $21.52 \pm 1.42 \mathrm{~b}$ & $24.17 \pm 1.20^{\mathrm{a}}$ & $75.7 \pm 3.1^{\mathrm{i}}$ & $18.39 \pm 1.82 \mathrm{a}$ & $7.443 \pm 1.104 \mathrm{a}$ & $0.813 \pm 0.017 \mathrm{~h}$ \\
\hline Bahçeli karası & $14.54 \pm 0.77^{1}$ & $13.22 \pm 0.67 \mathrm{k}$ & $13.20 \pm 0.72 \mathrm{k}$ & $13.64 \pm 0.67 \mathrm{~m}$ & $93.8 \pm 1.9^{b}$ & $5.86 \pm 0.58^{1}$ & $1.338 \pm 0.202^{1}$ & $0.874 \pm 0.012^{\mathrm{a}}$ \\
\hline Çavuş & $22.14 \pm 1.17 \mathrm{ef}$ & $20.10 \pm 1.24 \mathrm{~d}$ & $20.11 \pm 1.47 \mathrm{~d}$ & $20.76 \pm 1.22 \mathrm{e}$ & $93.7 \pm 2.4 \mathrm{~b}$ & $13.58 \pm 1.60 \mathrm{e}$ & $4.731 \pm 0.844 \mathrm{e}$ & $0.851 \pm 0.020 \mathrm{def}$ \\
\hline Cevşen & $20.54 \pm 0.69 \mathrm{hi}$ & $18.78 \pm 0.56^{\mathrm{f}}$ & $18.93 \pm 0.72 \mathrm{fg}$ & $19.40 \pm 0.53 \mathrm{fg}$ & $94.5 \pm 2.2 \mathrm{~b}$ & $11.83 \pm 0.64 \mathrm{fg}$ & $3.828 \pm 0.311 \mathrm{fg}$ & $0.859 \pm 0.016 \mathrm{bcd}$ \\
\hline Crimson & $20.73 \pm 1.27 \mathrm{~h}$ & $17.02 \pm 0.84 \mathrm{hi}$ & $17.16 \pm 0.92^{\mathrm{i}}$ & $18.22 \pm 0.90$ hij & $88.0 \pm 2.4 \mathrm{e}$ & $10.45 \pm 1.04 \mathrm{hi}$ & $3.189 \pm 0.479$ hi & $0.856 \pm 0.013$ cde \\
\hline Dimrit & $19.06 \pm 0.74^{j}$ & $17.34 \pm 0.72 \mathrm{hi}$ & $17.28 \pm 0.69^{\mathrm{i}}$ & $17.87 \pm 0.61^{\mathrm{j}}$ & $93.8 \pm 2.1 \mathrm{~b}$ & $10.04 \pm 0.68^{\mathrm{i}}$ & $2.997 \pm 0.307^{\mathrm{i}}$ & $0.858 \pm 0.014 \mathrm{bcd}$ \\
\hline Erenköy beyazı & $20.03 \pm 0.72^{\mathrm{i}}$ & $18.75 \pm 0.71 \mathrm{f}$ & $18.93 \pm 0.75 \mathrm{fg}$ & $19.23 \pm 0.67 \mathrm{~g}$ & $96.0 \pm 1.5^{a}$ & $11.63 \pm 0.82 \mathrm{~g}$ & $3.736 \pm 0.396 \mathrm{~g}$ & $0.851 \pm 0.016 \mathrm{def}$ \\
\hline Hafizali & $26.36 \pm 1.47^{b}$ & $20.53 \pm 0.95^{\mathrm{c}}$ & $20.92 \pm 1.00^{\mathrm{c}}$ & $22.45 \pm 1.00^{b}$ & $85.3 \pm 2.4^{\mathrm{f}}$ & $15.86 \pm 1.41 \mathrm{~b}$ & $5.957 \pm 0.790^{b}$ & $0.849 \pm 0.015$ ef \\
\hline Karaşabi & $22.68 \pm 1.22 \mathrm{de}$ & $18.23 \pm 0.87 \mathrm{~g}$ & $18.59 \pm 0.99 \mathrm{gh}$ & $19.73 \pm 0.88^{f}$ & $87.1 \pm 2.7 \mathrm{e}$ & $12.25 \pm 1.08^{\mathrm{f}}$ & $4.043 \pm 0.530^{\mathrm{f}}$ & $0.863 \pm 0.011 \mathrm{bc}$ \\
\hline Kırmızı & $21.42 \pm 1.17 \mathrm{~g}$ & $16.79 \pm 0.77^{\mathrm{i}}$ & $17.03 \pm 0.84^{\mathrm{i}}$ & $18.29 \pm 0.81 \mathrm{hi}$ & $85.5 \pm 2.5^{\mathrm{f}}$ & $10.53 \pm 0.95 \mathrm{hi}$ & $3.224 \pm 0.447 \mathrm{hi}$ & $0.855 \pm 0.013$ cde \\
\hline Izabella & $20.13 \pm 1.14^{\mathrm{i}}$ & $16.82 \pm 1.03^{\mathrm{i}}$ & $17.12 \pm 1.09^{\mathrm{i}}$ & $17.96 \pm 0.99 \mathrm{ij}$ & $89.3 \pm 2.7 \mathrm{~d}$ & $10.16 \pm 1.13 \mathrm{hi}$ & $3.059 \pm 0.518$ hi & $0.859 \pm 0.017 \mathrm{bcd}$ \\
\hline Morşabi & $23.08 \pm 1.18^{\mathrm{d}}$ & $18.24 \pm 0.9 \mathrm{~g}$ & $18.35 \pm 0.97 \mathrm{~h}$ & $19.76 \pm 0.90^{\mathrm{f}}$ & $85.7 \pm 2.4 \mathrm{f}$ & $12.29 \pm 1.12 \mathrm{f}$ & $4.065 \pm 0.552 \mathrm{f}$ & $0.852 \pm 0.013 \mathrm{def}$ \\
\hline Müşgüle & $22.32 \pm 0.99 \mathrm{e}$ & $19.49 \pm 0.68 \mathrm{e}$ & $19.65 \pm 0.74 \mathrm{e}$ & $20.44 \pm 0.66 \mathrm{e}^{\mathrm{e}}$ & $91.6 \pm 2.3^{c}$ & $13.14 \pm 0.85 \mathrm{e}$ & $4.485 \pm 0.440 \mathrm{e}$ & $0.852 \pm 0.019 \mathrm{def}$ \\
\hline Nuniya & $19.13 \pm 1.07^{\mathrm{j}}$ & $16.24 \pm 1.00 \mathrm{j}$ & $16.37 \pm 0.95^{j}$ & $17.19 \pm 0.90 \mathrm{k}$ & $89.9 \pm 2.5 \mathrm{~d}$ & $9.31 \pm 0.97^{j}$ & $2.681 \pm 0.418^{j}$ & $0.864 \pm 0.010^{b}$ \\
\hline Royal & $24.10 \pm 1.37^{c}$ & $21.99 \pm 1.30^{\mathrm{a}}$ & $21.99 \pm 1.34 \mathrm{a}$ & $22.66 \pm 1.19^{b}$ & $94.1 \pm 2.8^{b}$ & $16.18 \pm 1.72 \mathrm{~b}$ & $6.145 \pm 0.991 \mathrm{~b}$ & $0.872 \pm 0.012^{\mathrm{a}}$ \\
\hline Sultani çekirdeksiz & $16.37 \pm 0.75^{\mathrm{k}}$ & $12.36 \pm 0.45^{1}$ & $12.53 \pm 0.54^{1}$ & $13.63 \pm 0.42 \mathrm{~m}$ & $83.4 \pm 3.0 \mathrm{~g}$ & $5.84 \pm 0.36^{1}$ & $1.330 \pm 0.125^{1}$ & $0.836 \pm 0.020 \mathrm{~g}$ \\
\hline Yalova incisi & $26.69 \pm 1.76^{b}$ & $18.90 \pm 1.31^{f}$ & $19.20 \pm 1.41^{\mathrm{f}}$ & $21.31 \pm 1.35^{\mathrm{d}}$ & $79.9 \pm 2.9 \mathrm{~h}$ & $14.32 \pm 1.84 \mathrm{~d}$ & $5.125 \pm 0.994 \mathrm{~d}$ & $0.833 \pm 0.012 \mathrm{~g}$ \\
\hline Yerli beyaz & $16.71 \pm 0.80 \mathrm{k}$ & $15.84 \pm 0.67^{j}$ & $16.12 \pm 0.70 \mathrm{j}$ & $16.22 \pm 0.67^{1}$ & $97.1 \pm 1.5^{a}$ & $8.27 \pm 0.69 \mathrm{k}$ & $2.244 \pm 0.278 \mathrm{k}$ & $0.833 \pm 0.028 \mathrm{~g}$ \\
\hline Yuvarlak çekirdeksiz & $21.72 \pm 1.04 \mathrm{fg}$ & $16.75 \pm 0.64^{\mathrm{i}}$ & $17.24 \pm 0.72^{\mathrm{i}}$ & $18.44 \pm 0.67^{\mathrm{h}}$ & $85.0 \pm 2.5^{\mathrm{f}}$ & $10.69 \pm 0.78 \mathrm{~h}$ & $3.294 \pm 0.36^{\mathrm{h}}$ & $0.846 \pm 0.012 \mathrm{f}$ \\
\hline Mean \pm SD & $21.62 \pm 3.96$ & $17.96 \pm 2.57$ & $18.19 \pm 2.65$ & $19.16 \pm 2.82$ & $89.3 \pm 6.2$ & $11.78 \pm 3.36$ & $3.916 \pm 1.644$ & $0.852 \pm 0.021$ \\
\hline Min-max & $12.53-35.78$ & $11.49-25.54$ & $11.53-25.91$ & $12.37-26.44$ & $71.1-99.5$ & $4.81-21.96$ & $0.992-9.676$ & $0.769-0.890$ \\
\hline
\end{tabular}

*: Means followed by the same letter in the same column are not significantly different based on Duncan's test at $5 \%$ significance level.

\subsection{Eigen Statistics for Two Principal Components}

Results of principal component analysis are provided in Table 5. The first two principal components (PC1 and PC2) explained $99.5 \%$ of the total variation between the grape cultivars. PC1 had the greatest factor load. The factor loads for dimensional attributes were presented on $\mathrm{PC} 1$ and explained $78.5 \%$ of the variation between the grape cultivars. In factor-load-based ordering, the dimension variable with the greatest factor load was identified as thickness. It was remarkable that factor loads of dimensional variables (equivalent diameter, projected area and perimeter) measured at vertical orientation were greater than the values measured at horizontal orientation. PC2 explained $20.8 \%$ of the variation between the grape cultivars and the greatest factor loads were observed in elongation and sphericity variables. It was remarkable that there was a negative correlation between PC2 and sphericity. 
Table 5. Eigen statistics for two principal components.

\begin{tabular}{ccc}
\hline Variables & PC1 & PC2 \\
\hline Thickness & $\mathbf{0 . 9 9 9}$ & -0.006 \\
Feret diameter at vertical orientation. & $\mathbf{0 . 9 9 8}$ & -0.039 \\
Perimeter at vertical orientation. & $\mathbf{0 . 9 9 8}$ & -0.036 \\
Projected area at vertical orientation. & $\mathbf{0 . 9 9 7}$ & -0.032 \\
Width & $\mathbf{0 . 9 9 6}$ & -0.069 \\
Geometric mean diameter & $\mathbf{0 . 9 7 6}$ & 0.212 \\
Surface area & $\mathbf{0 . 9 6 8}$ & 0.247 \\
Volume & $\mathbf{0 . 9 5 1}$ & 0.280 \\
Feret diameter at horizontal orientation. & $\mathbf{0 . 9 4 0}$ & 0.337 \\
Perimeter at horizontal orientation. & $\mathbf{0 . 9 2 7}$ & 0.372 \\
Projected area at horizontal orientation. & $\mathbf{0 . 9 2 3}$ & 0.380 \\
Length & $\mathbf{0 . 8 1 1}$ & 0.583 \\
Aspect ratio at horizontal orientation. & 0.075 & $\mathbf{0 . 9 9 6}$ \\
Sphericity & $\mathbf{0 . 0 2 9}$ & -0.996 \\
Eigenvalues & 11.028 & 2.914 \\
Cum variance & 78.773 & 20.812 \\
\hline
\end{tabular}

In the scatter plot presented in Figure 4, dimensional variables had positive correlations with the PC1 axis. The greatest dimensions were seen in "Antep karasi", "Hafizali", "Royal" and "Amasya beyazi" cultivars. On the other hand, "Sultani çekirdeksiz" and "Bahçeli karasi" cultivars located on the left side of PC1 had the least dimensions. Since sphericity averages had negative correlations with the PC2 axis, the "Antep karasi" cultivar had the least sphericity value. The closest cultivars to each other in terms of both morphology and dimensional attributes were presented in a colored circle. For instance, "Müssgüle" and "Çavuş" cultivars had similar morphology and dimensional attributes. "Yuvarlak çekirdeksiz" and "Kırmızı" cultivars also had similar morphology and dimensional attributes. The morphology and dimension relationships of the cultivars or genotypes based on morphological characteristics are explained by multivariate statistical analysis methods. Morphological characterization is the first step for the description and classification of grape genotypes and the PCA method is a useful tool for screening the grape genotypes. The PCA method has been used to discriminate grape cultivars by using morphological, biochemical and even molecular data. PCA transforms the original variables into a limited number of uncorrelated new variables. The PCA method also allows the visualization of differences among individuals, the identification of groups and the identification of relationships among individuals and variables [55]. Lamine et al. [56] used the PCA method to discriminate Tunisian grape cultivars and reported high morphological diversity. Nassur et al. [57] used the PCA method on grape cultivars in Brazil, and based on principal component analysis (PCA), all grape cultivars were discriminated and high morphological variation was observed among the accessions. Istrate et al. [58] used applications of the principal component analysis (PCA) at grape varieties from the serogroup Coarnă neagră for establishing phenotypical variability and found great diversity among cultivars. Abiri et al. [55] determined high morphological and pomological variability of a grape (Vitis vinifera L.) germplasm collection in Iran by using PCA.

Thusly, in previous studies, differences in cultivars were put forth with principal component analysis in walnuts [42,59-61], kiwifruit [62], strawberry tree [63], hazelnuts [33], almonds [35], raspberry [64], citrus [65] and apricot [66]. All above studies indicated that PCA allows the extraction of the maximum information from used cultivars and underlining the interrelations between variables and individuals, either by similarity or opposition. 


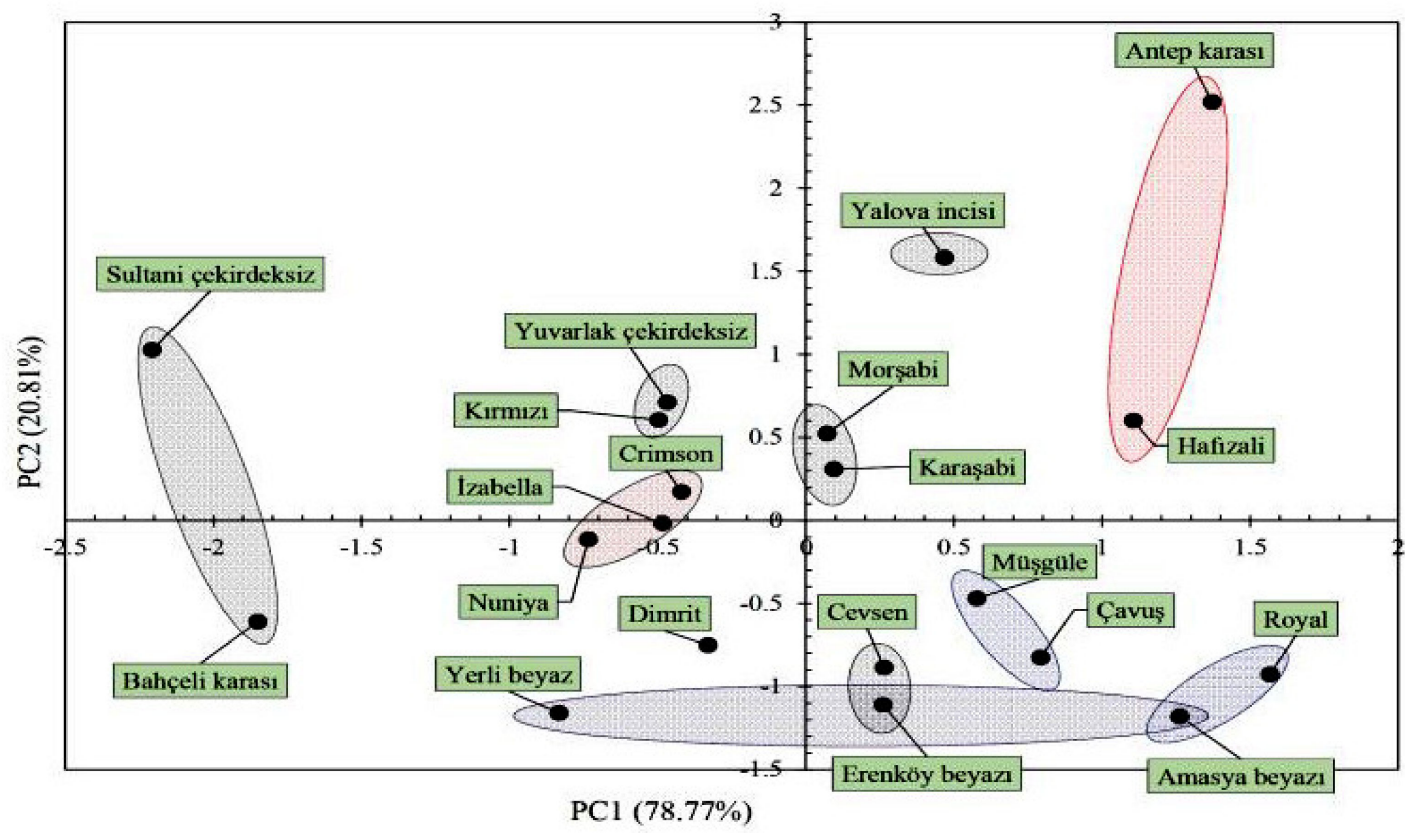

Figure 4. Scatter plot of principal component (PC) loadings defining morphology and dimensional attributes of the grape cultivars.

\subsection{The Results of the Discriminant Analysis and Pair-Wise Comparisons}

The contour codes obtained by Elliptic Fourier Analysis were subjected to principal component analysis (PCA), and morphology differences between the grape cultivars were explained by two principal components (Figure 5). The total variance explained was 94.56\%. PC1 explained $90.53 \%$ and PC2 explained $4.03 \%$ of the total variance. Considering the morphology differences explained by PC1, it was observed that ellipse and sphere geometries constituted the main source of variation. Fruit peduncle or widening at the base of the fruit constituted the source of variation explained by PC2. Grape berry looks similar to a water drop because of this widening. Relative oblateness on fruit surface constituted morphology variations of the genotypes.

$-2 \mathrm{SD} \quad$ Mean $\quad+2 \mathrm{SD}$
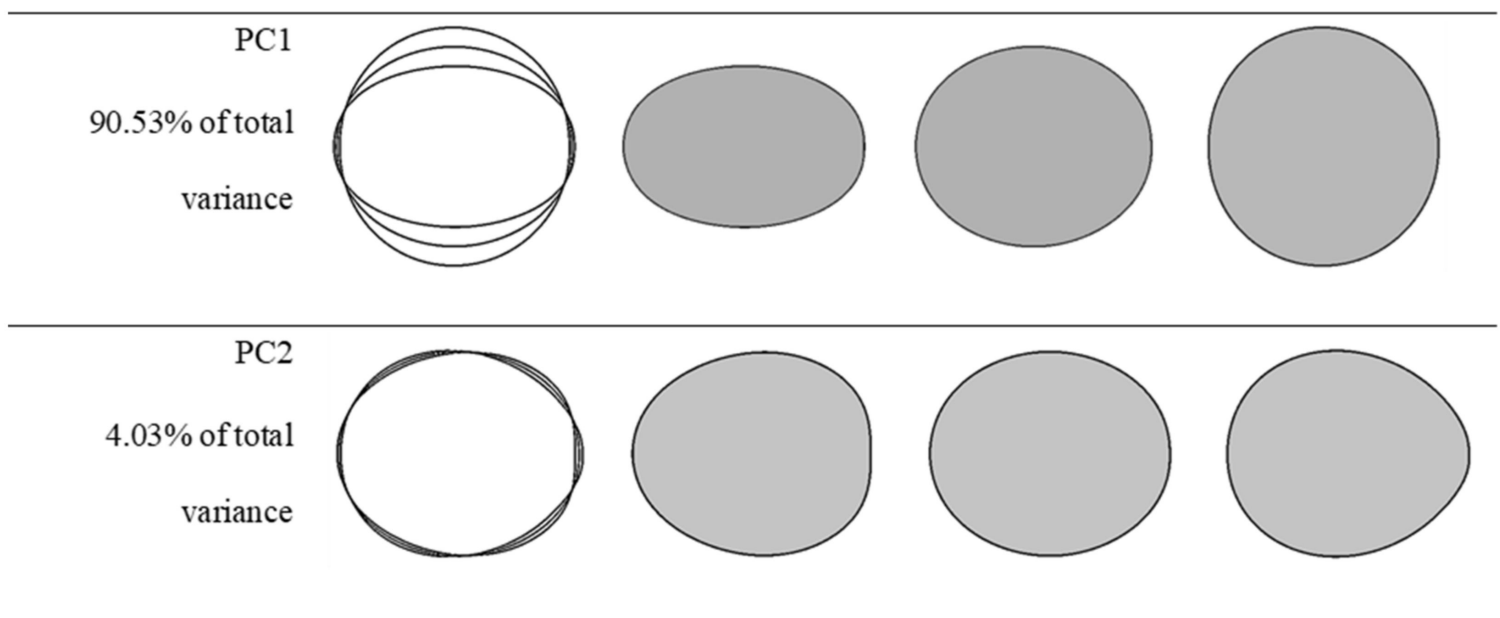

Figure 5. Principal components (PCs) as morphology variables of grape varieties based on a principal component analysis of 800 fruit outlines. From left to right, the outlines show the principal component scores corresponding to: mean -2 standard deviations, mean, mean +2 standard deviations. 
According to MANOVA results in Table 6, there were significant contour differences between the grape cultivars. In a linear discriminant analysis conducted with the use of component scores of EFA, two discriminant functions were obtained to discriminate grape contours from each other. The variance explained by the first and second discriminant functions was, respectively, identified as $81.5 \%$ and $18.5 \%$. In Hotelling's pair-wise comparison table, the grape cultivars indicated in color did not have significant morphology differences $(p>0.05)$. If the Mahalanobis distance value provided in pair-wise comparison is low, then the similarity between the cultivars is high. Similar findings were also reported by Demir et al. [38] for Cornus mas genotypes.

In Figure 6, the cultivars placed on the right side of the first discriminant function axis look similar to a sphere and the ones on the left side look similar to an ellipse. Peduncle connection section or fruit base is widened as moved away from the axis of the second discriminant function. The cultivars presented in frames in the graph were identified based on the results of the pair-wise comparison test. The cultivars placed in frames had close morphology to each other.

Results of cluster analysis conducted with the use of group centroids of discriminant functions are provided in Figure 7. Grape cultivars were separated into three morphological groups. Group I and II had six sub-groups. There is only one cultivar in group III.

Figure 7 indicated that the closest cultivars on the dendrogram were Dimrit and Royal, and cultivar Antep karası clearly differed from the rest of the cultivars in terms of berry morphology and dimensions. In fact, as indicated in Figure 1 and Table 4, Antep karasi has quite different fruit morphology and diameters than the other cultivars. Thus, group III, including Antep karasi, can be classified as out of the group. Previously, morphological data obtained from different grape cultivars showed different clustering patterns on dendrogram, and indicated that grape germplasm in different grapevine growing countries were quite variable in terms of morphological characteristics, which supports to our obtained result [54-57]. 
Table 6. The results of the discriminant analysis and pair-wise comparisons.

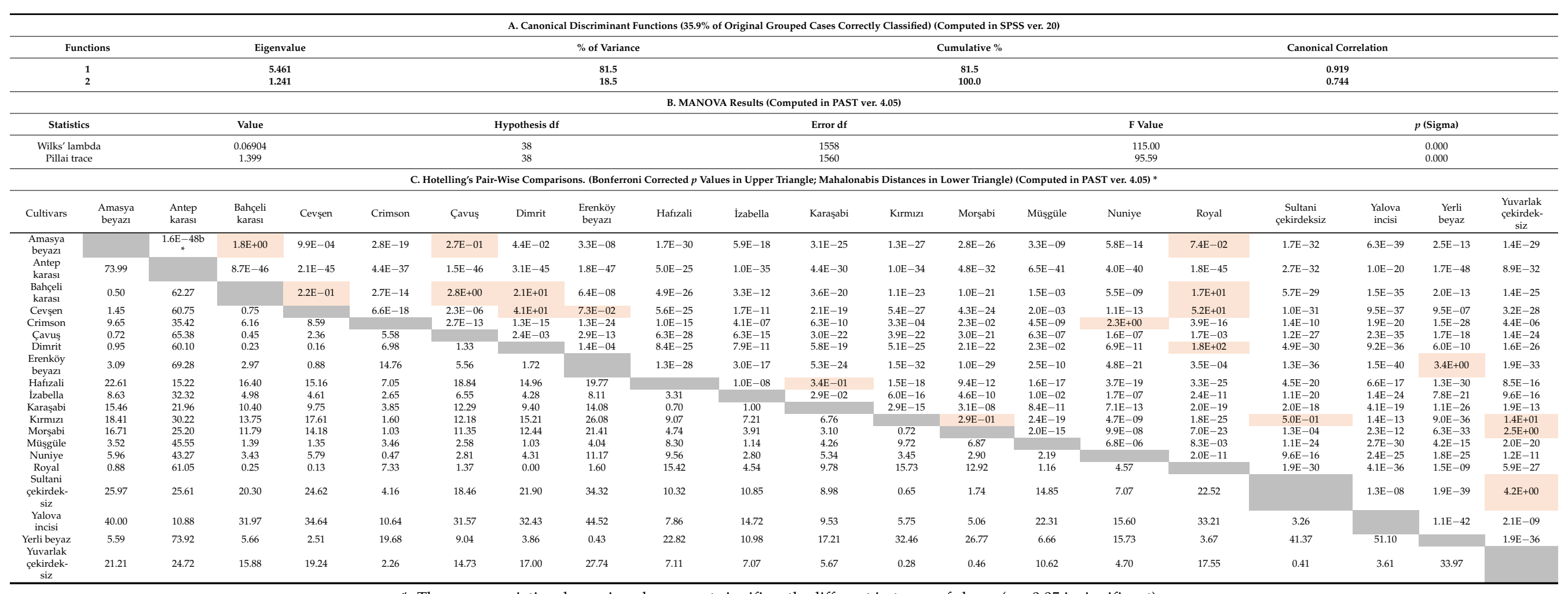

*: The grape varieties shown in color are not significantly different in terms of shape ( $p>0.05$ insignificant). 


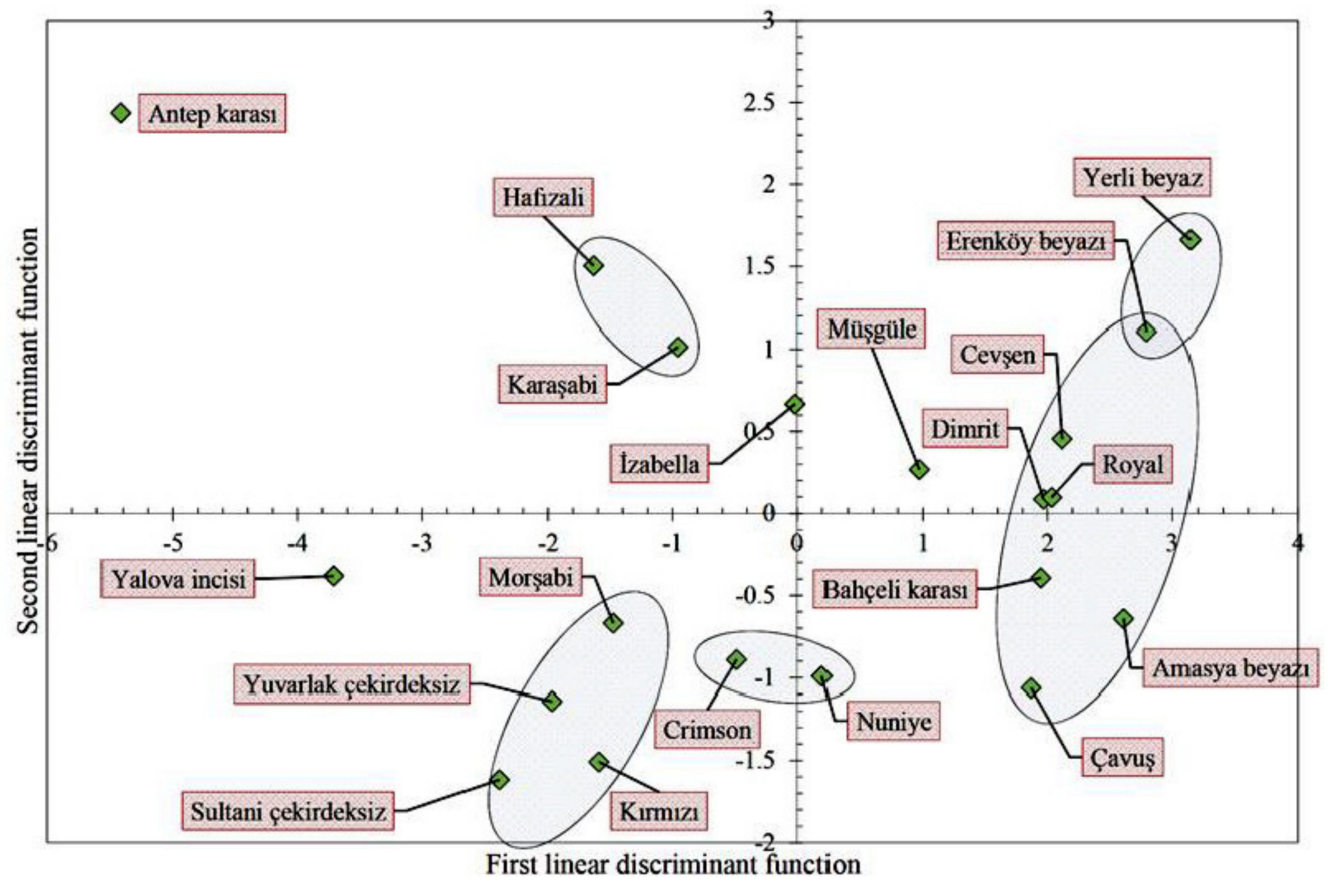

Figure 6. Biplot charts from linear discriminant analysis of 20 grape cultivars based on two principal component morphology variables derived from Elliptic Fourier data of 800 fruit outlines. (The locations of the cultivars on the chart show their own group centroid.)

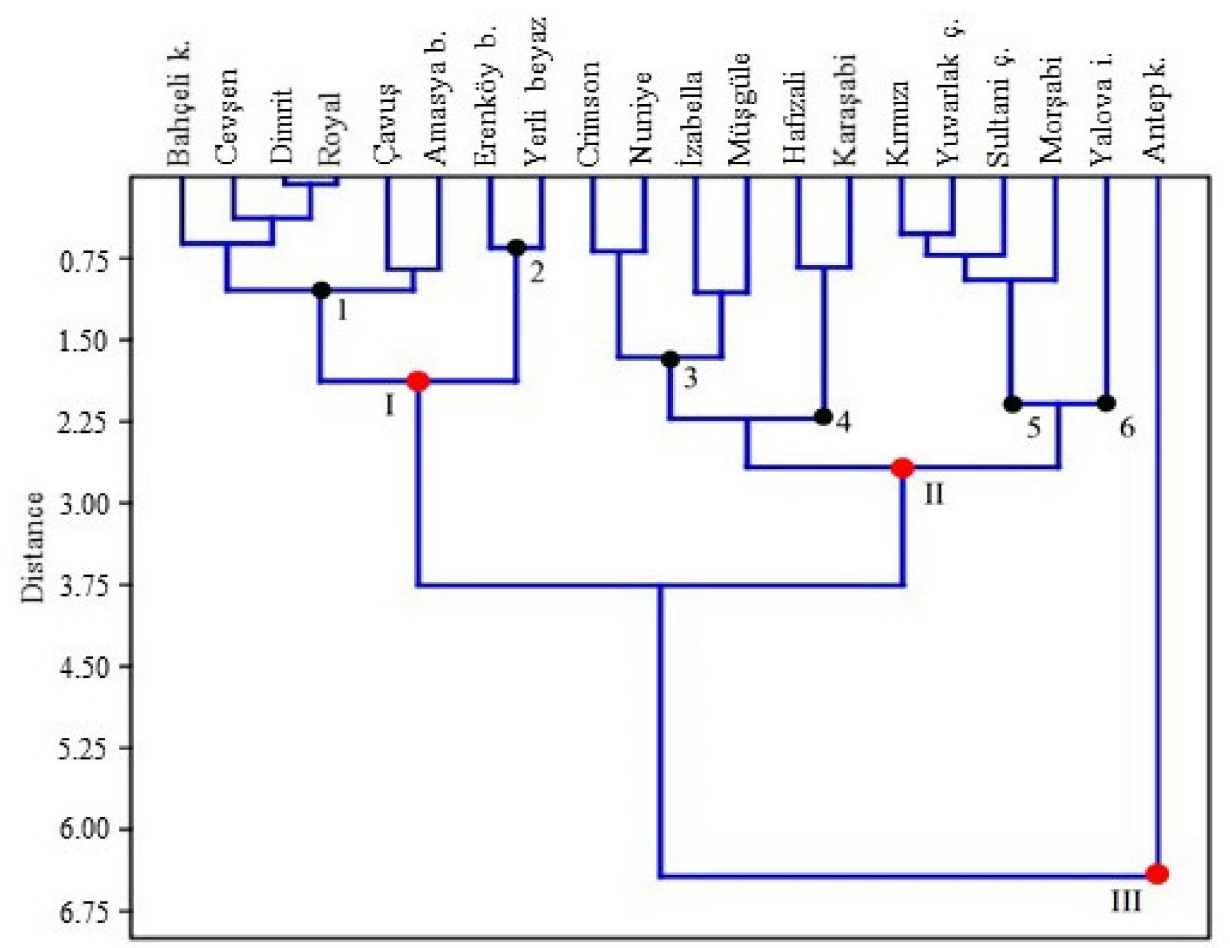

Figure 7. Dendrogram of 20 grape cultivars for the first two principal component scores using hierarchical cluster analysis (between-group linkage method and Euclidean distance). 


\section{Conclusions}

The projected area, equivalent diameter and perimeter values measured at horizontal orientation were greater than the values measured at vertical orientation. Elongation ratio averages of 20 grape cultivars are not dependent on diameter, area and perimeter of the cultivars. Elongation averages of the cultivars exhibited large variations. The greatest length-to-width ratio was 1.56 . There were some cultivars with a morphology quite close to the sphere. When the geometrical dimensions of the cultivars with a high sphericity average were assessed, it was observed that they were both large and small sizes. Principal component analysis revealed that present grape cultivars were distinguished from each other based on dimensional characteristics rather than morphology. Dimensional variables constituted the most significant source of variation between the grape cultivars. The most significant shape variables explaining the variation between the cultivars were identified as sphericity and elongation.

Elliptic Fourier analysis revealed that present grape cultivars looked similar to an ellipse or sphere in morphology. There are also some cultivars that looked similar to a water drop because of widening at the peduncle or fruit base. Linear discriminant analysis and cluster analysis clearly demonstrated similarities between the grape cultivars. In terms of morphology, grape cultivars were classified into $7 \mathrm{sub}$-groups. The first sub-group included "Bahçeli karası", "Cevşen", "Dimrit", "Royal", "Çavuş" and "Amasya beyazı" cultivars; the second group included "Erenköy beyaz1" and "Yerli beyaz" cultivars; the third group included "Crimson", "Nuniya", "İzabella" and "Müşgüle" cultivars; the fourth sub-group included "Hafızali" and "Karaşabi" cultivars; the fifth sub-group included "Kırmızı", "Yuvarlak çekirdeksiz", "Sultani çekirdeksiz" and "Morşabi" cultivars; the sixth sub-group included "Yalova incisi" and the seventh sub-group included the "Antep karasi" cultivar.

Author Contributions: Conceptualization, B.S. and M.K.; data curation, B.D. and M.K; formal analysis, S.E., B.S. and B.D.; methodology, B.S. and B.D.; project administration, M.K. and S.E.; visualization, B.S., M.B., J.S and B.D.; writing-original draft, S.E., M.B., B.S., B.D., M.K. and J.S.; writing-review and editing, S.E., M.B. and J.S. All authors have read and agreed to the published version of the manuscript.

Funding: This study was carried out in the Mersin University Advanced Technology Education Research and Application Center (MEITAM) Image Processing Laboratory, and by the project CZ.02.1.01/0.0/0.0/16_017/0002334 Research Infrastructure for Young Scientists; this is co-financed by Operational Programme Research, Development and Education.

Institutional Review Board Statement: Not applicable.

Informed Consent Statement: Not applicable.

Data Availability Statement: All-new research data were presented in this contribution.

Conflicts of Interest: The authors declare that they have no conflict of interest.

\section{References}

1. FAOSTAT. FAO Web Page. Available online: http:/ / www.fao.org/faostat (accessed on 15 May 2020).

2. OIV. Statistical Report on World Vitiviniculture. 2019. Available online: https://www.oiv.int/public/medias /6782/oiv-2019 -statistical-report-on-world-vitiviniculture.pdf (accessed on 5 June 2021).

3. OIV. Distribution of the World's Grapevine Varieties. 2017. Available online: https://www.oiv.int/public/medias/5888/endistribution-of-the-worlds-grapevine-varieties.pdf (accessed on 5 June 2021).

4. Agaoglu, Y.S.; Celik, H.; Gokcay, E. Brief Ampelographic Characterization of Indigenous Grapevine Cultivars Subjected to Clonal Selection in Turkey. In Proceedings of the 5th International Symposium on Grape Breeding, St. Martin/Pfalz, Germany, 12-16 September 1989.

5. Maghradze, D.; Kikilashvili, S.; Gotsiridze, O.; Maghradze, T.; Fracassetti, D.; Failla, O.; Rustioni, L. Comparison between the grape technological characteristics of Vitis vinifera Subsp. sylvestris and Subsp. sativa. Agronomy 2021, 11, 472. [CrossRef]

6. Bouby, L.; Wales, N.; Jalabadze, M.; Rusishvili, N.; Bonhomme, V.; Ramos-Madrigal, J.; Evin, A.; Ivorra, S.; Lacombe, T.; Pagnoux, C.; et al. Tracking the history of grapevine cultivation in Georgia by combining geometric morphometric and ancient DNA. Veget. Hist. Archaeobot. 2020, 30, 63-76. [CrossRef] 
7. Soylemezoglu, G.; Agaoglu, Y.S.; Uzun, H.I. Ampelographic characteristics and isozymic analysis of Vitis vinifera spp. sylvestris Gmel in Southwestern Turkey. Biotechno. Biotechnol. Equip. 2001, 15, 106-113. [CrossRef]

8. Soylemezoglu, G.; Atak, A.; Boz, Y.; Unal, A.; Saglam, M. Viticulture in Turkey. Chron. Horticult. 2016, 56, $27-31$.

9. Ates, F.; Coban, H.; Kara, Z.; Sabir, A. Ampelographic characterization of some grape cultivars (Vitis vinifera L.) grown in south-western region of Turkey. Bulg. J. Agric. Sci. 2011, 17, 314-324.

10. Eyduran, S.P.; Akin, M.; Ercisli, S.; Eyduran, E.; Magharadze, D. Sugars, organic acids, and phenolic compounds of ancient grape cultivars (Vitis vinifera L.) from Igdir province of Eastern Turkey. Biol. Res. 2015, 48, 2. [CrossRef] [PubMed]

11. Sabir, A.; Kafkas, E.; Tangolar, S. Distribution of major sugars, acids and total phenols in juice of five grapevine (Vitis spp.) cultivars at different stages of berry development. Span. J. Agric. Res. 2010, 8, 425-433. [CrossRef]

12. Pallas, E.I. Sustainable wine and grape production, the example of Hungary. Visegrad J. Bioeconomy Sustain. Develop. 2016, 5, 53-57. [CrossRef]

13. Sargolzaei, M.; Rustioni, L.; Cola, G.; Ricciardi, V.; Bianco, P.A.; Maghradze, D.; Failla, O.; Quaglino, F.; Toffolatti, S.L.; de Lorenzis, G. Georgian Grapevine Cultivars: Ancient Biodiversity for Future Viticulture. Front. Plant Sci. 2021, 12, 630122. [CrossRef]

14. Kupe, M. Some ampelographic and biochemical characteristics of local grape accessions from Turkey. Genetika-Belgrade 2020, 50, 513-525. [CrossRef]

15. Gago, P.; Santiago, J.L.; Boso, S.; Alonso-Villaverde, V.; Grando, M.S.; Martinez, M.C. Biodiversity and characterization of twenty-two Vitis vinifera L. cultivars in the Northwestern Iberian Peninsula. Am. J. Enol. Vitic. 2009, 60, $293-301$.

16. Antolín, M.C.; Toledo, M.; Pascual, I.; Irigoyen, J.J.; Goicoechea, N. The exploitation of local Vitis vinifera L. Biodiversity as a valuable tool to cope with climate change maintaining berry quality. Plants 2021, 10, 71. [CrossRef]

17. Meneghetti, S.; Costacurta, A.; Frare, E.; da Rold, G.; Migliaro, D.; Morreale, G.; Crespan, M.; Sotés, V.; Calò, A. Clones identification and genetic characterization of Garnacha grapevine by means of different PCR-derived marker systems. Mol. Biothecnol. 2011, 48, 244-254. [CrossRef] [PubMed]

18. Pantelic, M.; Zagorac, D.D.; Natic, M.; Gašic, U.; Jovic, S.; Vujovic, D.; Djordjevic, P. Impact of clonal variability on phenolics and radical scavenging activity of grapes and wines: A study on the recently developed Merlot and Cabernet Franc clones (Vitis vinifera L.). PLoS ONE 2016, 11, e0163823. [CrossRef] [PubMed]

19. OIV. OIV Descriptor List for Grape Varieties and Vitis Species, 2nd ed.; OIV: Paris, France, 2001.

20. Muñoz-Organero; Gaforio, L.; Garcia Muñoz, S.; Cabello, F. Manual for Standarization of Vitis Descriptors; Instituto Nacional de Investigación y Tecnología Agraria y Alimentaria (INIA): Madrid, Spain, 2011.

21. Rustioni, L.; Maghradze, D.; Popescu, C.F.; Cola, G.; Abashidze, E.; Aroutiounian, R.; Brazao, J.; Coletti, S.; Cornea, V.; Dejeu, L.; et al. First results of the European grapevine collections' collaborative network: Validation of a standard eno-carpological phenotyping method. Vitis-Geilweilerhof. 2014, 53, 219-226.

22. Rustioni, L.; Cola, G.; Maghradze, D.; Abashidze, E.; Argiriou, A.; Aroutiounian, R.; Brazão, J.; Chipashvili, R.; Cocco, M.; Cornea, V.; et al. Description of the Vitis vinifera L. phenotypic variability in eno-carpological traits by a Euro-Asiatic collaborative network among ampelographic collections. Vitis Geilweilerhof. 2019, 58, 37-46.

23. Mena, A.; Martínez, J.; Fernández-González, M. Recovery, identification and relationships by microsatellite analysis of ancient grapevine cultivars from Castilla-La Mancha: The largest wine growing region in the world. Genet. Resour. Crop Evol. 2014, 61, 625-637. [CrossRef]

24. Benjak, A.; Ercisli, S.; Vokurka, A.; Maletic, E.; Pejic, I. Genetic relationships among grapevine cultivars native to Croatia, Greece and Turkey. Vitis 2005, 44, 73-77.

25. Laiadi, Z.; Bentchikou, M.M.; Bravo, G.; Cabello, F.; Martínez-Zapater, J.M. Molecular identification and genetic relationships of Algerian grapevine cultivars maintained at the germplasm collection of Skidda (Algeria). Vitis 2009, 48, 25-32.

26. García-Muñoz, S.; Lacombe, T.; de Andrés, M.T.; Gaforio, L.; Muñoz-Organero, G.; Lacou, V.; This, P.; Cabello, F. Grape varieties (Vitis vinifera L.) from the Balearic Islands: Genetic characterization and relationship with Iberian peninsula and Mediterranean Basin. Genet. Resour. Crop Evol. 2012, 59, 589-605. [CrossRef]

27. Maeda, H.; Akagi, T.; Tao, R. Quantitative characterization of fruit shape and its differentiation pattern in diverse persimmon (Diospyros kaki) cultivars. Sci. Hortic. 2018, 228, 41-48. [CrossRef]

28. Basile, T.; Marsico, A.D.; Perniola, R. NIR analysis of intact berries: Chemical and physical properties prediction using multivariate analysis. Foods 2021, 10, 113. [CrossRef]

29. Fuantes, S.; Hernandez-Montes, E.; Escalona, J.M.; Bota, J.; Gonzales Viejo, C.; Poblete-Echeverria, C.; Tongson, E.; Medrano, H. Automated grapevine cultivar classification based on machine learning using leaf morpho-colorimetry, fractal dimension and near-infrared spectroscopy parameters. Comput. Electron. Agrc. 2018, 151, 311-318. [CrossRef]

30. De Oliveira, G.L.; de Souza, A.P.; de Oliveira, F.A.; Zucchi, M.I.; de Souza, L.M.; Moura, M.F. Genetic structure and molecular diversity of Brazilian grapevine germplasm: Management and use in breeding programs. PLoS ONE 2020, 15, e0240665. [CrossRef]

31. Laucou, V.; Launay, A.; Bacilieri, R.; Lacombe, T.; Adam-Blondon, A.F.; Bérard, A.; Chauveau, A.; de Andrés, M.T.; Hausmann, L.; Ibanez, J.; et al. Extended diversity analysis of cultivated grapevine Vitis vinifera with 10K genome-wide SNPs. PLoS ONE 2018, 13, e0192540. [CrossRef] [PubMed]

32. Bodor, P.; Somogyi, E.; Baranyai, L.; Lazar, J.; Balo, B. Analysis of the grapevine (Vitis vinifera L.) berry shape by using elliptic Fourier descriptors. Prog. Agric. Eng. Sci. 2020, 16, 87-93. 
33. Sayıncı, B.; Kara, M.; Ercişli, S.; Duyar, Ö.; Ertürk, Y. Elliptic Fourier analysis for shape distinction of Turkish hazelnut cultivars. Erwerbs Obstbau 2015, 57, 1-11. [CrossRef]

34. Shimomura, K.; Horie, H.; Sugiyama, M.; Kawazu, Y.; Yoshioka, Y. Quantitative evaluation of cucumber fruit texture and shape traits reveals extensive diversity and differentiation. Sci. Hortic. 2016, 199, 133-141. [CrossRef]

35. Demir, B.; Sayinci, B.; Cetin, N.; Yaman, M.; Comlek, R. Shape discrimination of almond cultivars by Elliptic Fourier Descriptors. Erwerbs Obstbau. 2018, 61, 245-256. [CrossRef]

36. Iwata, H.; Ukai, Y. SHAPE: A computer program package for quantitative evaluation of biological shapes based on elliptic Fourier descriptors. J. Hered. 2002, 93, 384-385. [CrossRef]

37. Kara, M.; Sayınc1, B.; Elkoca, E.; Öztürk, İ; Özmen, T.B. Seed size and shape analysis of registered common bean (Phaseolus vulgaris L.) cultivars in Turkey using digital photography. J. Agric. Sci. 2013, 19, 219-234.

38. Demir, B.; Sayinci, B.; Sümbül, A.; Yaman, M.; Yildiz, E.; Çetin, N.; Karakaya, O.; Ercişli, S. Bioactive compounds and physical attributes of Cornus mas genotypes through multivariate approaches. Folia Hortic. 2020, 32, 189-202. [CrossRef]

39. Kara, M. Physical Properties of Biological Products (Includes Agricultural Products and Foods); Güven®Bilimsel I. Press: İzmir, Turkey, 2017; p. 327, (In Turkish). ISBN 978-975-6240-56-4.

40. Mohsenin, N.N. Physical Properties of Plant and Animal Materials; Gordon and Breach Science Publisher: New York, NY, USA, 1986.

41. Sayınc1, B.; Ercişli, S.; Akbulut, M.; Şavşatlı, Y.; Baykal, H. Determination of shape in fruits of cherry laurel (Prunus laurocerasus) accessions by using Elliptic Fourier analysis. Acta Sci. Pol. Hortoru 2015, 14, 63-82.

42. Ercisli, S.; Sayinci, B.; Kara, M.; Yildiz, C.; Ozturk, I. Determination of size and shape features of walnut (Juglans regia L.) cultivars using image processing. Sci Hortic. 2012, 133, 47-55. [CrossRef]

43. Sayınc1, B. Detection of manufacturing defects on orifice geometry of polyacetal (POM) nozzle discs by using the elliptic fourier descriptors. J. Agric. Fac. Bursa Uludă̆ Univ. 2016, 30, 57-73.

44. Neto, J.C.; Meyer, G.E.; Jones, D.D.; Samal, A.K. Plant species identification using Elliptic Fourier leaf shape analysis. Comput. Electron. Agric. 2006, 50, 121-134. [CrossRef]

45. Özkan-Koca, A. Ortadoğu'da yayılış gösteren Apis mellifera L. (Hymenoptera: Apidae) alt türlerinin geometrik morfometri yöntemiyle analizi. Ph.D. Thesis, Ankara Üniversitesi Fen Bilimleri Enstitüsü, Biyoloji Anabilim Dalı, Ankara, Turkey, 2012.

46. Ekhvaia, J.; Akhalkatsi, M. Morphological variation and relationships of Georgian populations of Vitis vinifera L. subsp. sylvestris (C.C. Gmel.). Flora 2010, 205, 608-617. [CrossRef]

47. Leão, P.C.S.; Cruz, C.D.; Motoike, S.Y. Genetic diversity of table grape based on morphoagronomic traits. Sci. Agric. 2011, $68,42-49$. [CrossRef]

48. Khadivi-Khub, A.; Salimpour, A.; Rasouli, M. Analysis of grape germplasm from Iran based on fruit characteristics. Braz. J. Bot. 2014, 37, 105-113. [CrossRef]

49. Kök, D.; Bal, E.; Bahar, E. Physical and biochemical traits of selected grape varieties cultivated in Tekirdağ, Turkey. Int. J. Sustain. Agric. Manag. Inform. 2017, 3, 215-223. [CrossRef]

50. Abu-Zahra, T. Berry size of Thompson seedless as influenced by the application of Gibberellic acid and cane girdling. Pak. J. Bot. 2010, 42, 1755-1760.

51. Barbagallo, M.G.; Guidoni, S.; Hunter, J.J. Berry size and qualitative characteristics of Vitis vinifera L. cv. Syrah. S. Afr. J. Enol. Vitic. 2011, 32, 129-136. [CrossRef]

52. Kose, B. Effect of rootstock on grafted grapevine quality. Eur. J. Hortic. Sci. 2014, 79, 197-202.

53. Esgici, R.; Özdemir, G.; Pekitkan, G.; Eliçin, K.; Öztürk, F.; Sessiz, A. Engineering properties of the Şire grape (Vitis vinifera L. Cv.). Sci. Papers Ser. B Hortic. 2017, 61, 195-203.

54. Khodaei, J.; Akhijahani, H.S. Some physical properties of rasa grape (Vitis vinifera L.). World Appl. Sci. J. 2012, 18, 818-825.

55. Abiri, K.; Rezaei, M.; Tahanian, H.; Heidari, P.; Khadivi, A. Morphological and pomological variability of a grape (Vitis vinifera L.) germplasm collection. Sci. Hortic. 2020, 266, 109285. [CrossRef]

56. Lamine, M.; Zemni, H.; Ziadi, S.; Chabaane, A.; Melki, I.; Mejri, S.; Zoghlami, N. Multivariate analysis and clustering reveal high morphological diversity in Tunisian autochthonous grapes (Vitis vinifera): Insights into characterization, conservation and commercialization. J. Int. Sci. Vigne. Vin. 2014, 48, 111-122. [CrossRef]

57. Nassur, R.C.M.R.; Pereira, G.E.; Alves, J.A.; Lima, L.C.O. Chemical characteristics of grape juices from different cultivar and rootstock combinations. Pesq. Agropec. Bras. 2014, 49, 540-545. [CrossRef]

58. Istrate, A.; Rotaru, L.; Colibaba, L.C. Applications of the principle component analysis (PCA) at grape varieties from the sortogroup Coarnă neagră for establishing phenotypical variability. Seria Hortic. 2015, 58, 163-168.

59. Demir, B.; Sayıncı, B.; Çetin, N.; Yaman, M.; Çömlek, R.; Aydın, Y.; Sütyemez, M. Elliptic Fourier based analysis and multivariate approaches for size and shape distinctions of walnut (Juglans regia L.) cultivars. Grasas Aceites 2018, 69, 1-12. [CrossRef]

60. Skender, A.; Kurtovic, M.; Drkenda, P.; Becirspahic, D.; Ebrahimi, A. Phenotypic variability of autochthonous walnut (Juglans regia L) genotypes in northwestern Bosnia and Herzegovina. Turk. J. Agric. For. 2020, 44, 517-525. [CrossRef]

61. Bujdosó, G.; Cseke, K. The Persian (English) walnut (Juglans regia L.) assortment of Hungary: Nut characteristics and origin. Sci. Hortic. 2021, 283, 110035. [CrossRef]

62. Ercisli, S.; Esitken, A.; Cangi, R.; Sahin, F. Adventitious root formation of kiwifruit in relation to sampling date, IBA and Agrobacterium rubi inoculation. Plant Growth Regul. 2003, 41, 133-137. [CrossRef] 
63. Serçe, S.; Özgen, M.; Torun, A.A.; Ercisli, S. Chemical composition, antioxidant activities and total phenolic content of Arbutus andrachne L. (Fam. Ericaceae) (the Greek strawberry tree) fruits from Turkey. Anal. J. Food Compos. 2010, 23, 619-623. [CrossRef]

64. Zivotic, A.; Micic, N.; Zabic, M.; Bosancic, B.; Cvetkovic, M. Precision cane meristem management can influence productivity and fruit quality of floricane red raspberry cultivars. Turk. J. Agric. For. 2019, 43, 405-413. [CrossRef]

65. Sulu, G.; Kacar, Y.; Polat, I.; Kitapci, A.; Turgutoglu, E.; Simsek, O.; Satar, G. Identification of genetic diversity among mutant lemon and mandarin varieties using different molecular markers. Turk. J. Agric. For. 2020, 44, 465-478. [CrossRef]

66. Gecer, M.K.; Kan, T.; Gundogdu, M.; Ercisli, S.; Ilhan, G.; Sagbas, H.I. Physicochemical characteristics of wild and cultivated apricots (Prunus armeniaca L.) from Aras valley in Turkey. Genet. Resour. Crop Evol. 2020, 67, 935-945. [CrossRef] 